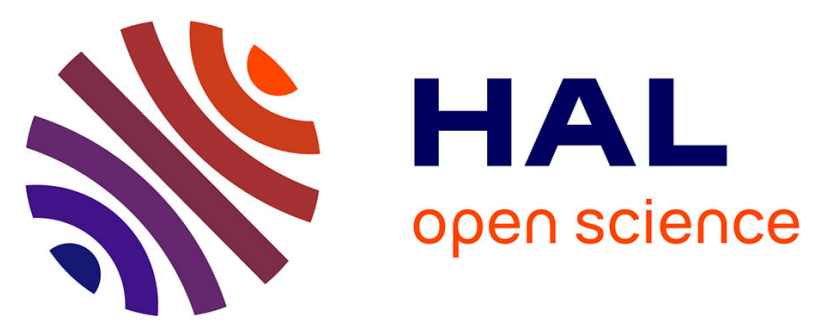

\title{
Surface manipulation of a curved polycyclic aromatic hydrocarbon-based nano-vehicle molecule equipped with triptycene wheels
}

We -Hyo Soe, Corentin Durand, Olivier Guillermet, Sebastien Gauthier, Henri-Pierre Jacquot de Rouville, Saurabh Srivastava, Claire Kammerer, Gwénaël Rapenne, Christian Joachim

\section{To cite this version:}

We -Hyo Soe, Corentin Durand, Olivier Guillermet, Sebastien Gauthier, Henri-Pierre Jacquot de Rouville, et al.. Surface manipulation of a curved polycyclic aromatic hydrocarbon-based nano-vehicle molecule equipped with triptycene wheels. Nanotechnology, 2018, 29 (49), pp.495401. 10.1088/13616528/aae0d9 . hal-01908676

\section{HAL Id: hal-01908676 https://hal.science/hal-01908676}

Submitted on 10 May 2020

HAL is a multi-disciplinary open access archive for the deposit and dissemination of scientific research documents, whether they are published or not. The documents may come from teaching and research institutions in France or abroad, or from public or private research centers.
L'archive ouverte pluridisciplinaire HAL, est destinée au dépôt et à la diffusion de documents scientifiques de niveau recherche, publiés ou non, émanant des établissements d'enseignement et de recherche français ou étrangers, des laboratoires publics ou privés. 


\title{
Surface manipulation of a curved polycyclic aromatic hydrocarbon-based nanovehicle molecule equipped with triptycene wheels
}

\author{
W.-H. Soe ${ }^{\mathrm{a}, \mathrm{b}}$, C. Durand ${ }^{\mathrm{a}}$, O. Guillermet ${ }^{\mathrm{a}}$, S. Gauthier ${ }^{\mathrm{a}}$, H.-P. Jacquot de Rouville ${ }^{\mathrm{a}}$, S. \\ Srivastava $^{\mathrm{a}, \mathrm{b}}$, C. Kammerer ${ }^{\mathrm{a}}$, G. Rapenne ${ }^{\mathrm{a}, \mathrm{c}}$ and C. Joachim ${ }^{\mathrm{a}, \mathrm{b}}$
}

${ }^{a}$ CEMES, Université de Toulouse, CNRS,

29 Rue J. Marvig, BP 94347, 31055 Toulouse Cedex, France

${ }^{b}$ International Center for Materials Nanoarchitectonics (WPI-MANA),

National Institute for Materials Science (NIMS),

1-1 Namiki, Tsukuba, Ibaraki 305-0044, Japan

${ }^{\mathrm{c}}$ Division of Materials Science, Nara Institute of Science and Technology, 8916-5 Takayama, Ikoma, Nara, Japan 


\begin{abstract}
With a central curved chassis, a four wheels molecule-vehicle was deposited on an $\mathrm{Au}(111)$ surface and imaged at low temperature using a scanning tunneling microscope. The curved conformation of the chassis and the consequent moderate interactions of the four wheels with the surface were observed. The $\mathrm{dI} / \mathrm{dV}$ constant current maps of the tunneling electronic resonances close to the $\mathrm{Au}(111)$ Fermi level were recorded to identify the potential energy entry port on the molecular skeleton to trigger and control a driving of the molecule. A lateral pushing mode of molecular manipulation and the consequent recording of the manipulation signals confirm how the wheels can step by step rotate while passing over the Au(111) surface native herringbone reconstructions. Switching a phenyl holding a wheel to the chassis was not observed for triggering a lateral molecular motion inelastically and without any mechanic push by the tip apex. This points out the necessity to encode the sequence of the required wheels action on the profile of potential energy surface of the excited states to be able to drive a molecule-vehicle.
\end{abstract}




\section{1) Introduction}

Single molecule nano-vehicles are a new class of organic molecules generally equipped with molecular legs, paddles or wheels chemical groups and able to be controllably driven on a surface with an atomic scale precision [1]. After their first complete design and synthesis with 2 legs \& 2 wheels [2,3] and 4 wheels [4] at the beginning of the century, a large variety of molecule nano-vehicles have been proposed, synthesized and tested for example with two Binaphthyl paddles [5], or with a variety of molecular wheels like the four triptycene molecular group used bellow $[6,7,8]$ or with four switchable legs [9].

Molecule nano-vehicles are expected to cargo an atomic scale load from one to another a specific location on a surface [10]. Their molecular skeleton is generally made of a central (rigid) planar molecular structure (called the chassis) where the molecular legs, paddle or wheels are chemically bound $[1,2,4]$. This chassis is supposed to be maintained at van der Waals distances away from the surface to minimize the lateral diffusion potential energy barrier of the molecule nano-vehicle on its supporting surface (or at least to make this barrier only dependent on its holders (legs, paddles, wheels)). Those holders must also ensure at least a reproducible step by step displacement of the nano-vehicle on this surface. For this displacement to be controllable, the nano-vehicle motive power can be delivered by a molecular motor chemical group directly embedded in the chemical structure of the chassis (apparent [11] or quantum $[5,12])$ or by the holder themselves when they are well synchronized together to achieve a controllable lateral motion on the surface [9].

We report here the surface deposition, LT-UHV-STM images recording, $\mathrm{d} / \mathrm{dV}$ mapping and single molecule manipulations of a four wheels molecule nano-vehicle equipped with a curved chassis. By its peculiar intramolecular mechanics, the concept of curved chassis was introduced by some of us [13] to moderate the interactions between the wheels and the supporting surface. Certain molecular wheels like $\mathrm{C}_{60}[6]$ or carborane [14] are known to lead to a very small lateral diffusion potential energy barrier for example on the $\mathrm{Au}(111)$ surface. In this case, the nanovehicle lateral motion is very difficult to control unless a more reactive supporting surface is selected to compensate for this small lateral diffusion barrier [15]. This can be a problem for nano-vehicles carrying a load because the load itself can be in this case also chemisorbed. In another context, the "pacman" molecule is a good example of a loadable molecule whose lateral diffusion barrier becomes so large that the molecule and its atoms cargo were not moving at all after loading more than 5 atoms [16]. On the contrary, other wheels like triptycenes present a 
large lateral diffusion barrier (mounted on a flat chassis [17]). Only STM molecular mechanical manipulation in a strong repulsive regime of mechanical interactions with the STM tip can succeed in putting it in motion laterally on the supporting surface, unless a well-adapted chassis is used to minimize the individual triptycene interactions with this metal surface [18].

The polycyclic aromatic hydrocarbon-based chassis equipped with four triptycene wheels (PAH-4W) shown in Fig. 1 was synthesized [13] with the goal to test how the curvature of this molecule favors or not the triptycene wheels rotation when physisorbed on an $\mathrm{Au}(111)$ surface. We present in this paper our experimental conclusions on this chassis curvature effect. In section 2, the flash sublimation, the LT-UHV-STM images and the corresponding $\mathrm{dI} / \mathrm{dV}$ mapping of the PAH-4W molecule are presented. In section 3, the LT-UHV-STM molecular mechanical manipulations are discussed together with our attempt, when one wheel appears to be up on the $\mathrm{Au}(111)$ surface, to drive the PAH-4W by tunneling inelastic effect just at the threshold of dismounting a wheel from the chassis. In section 4, the LT-UHV-STM manipulation results are discussed in terms of intramolecular mechanics taking into account the different molecular conformations observed on the $\mathrm{Au}(111)$ surface. The PAH-4W molecule was engaged by the Toulouse team in the first international 2017 nanocar race [1,19].

\section{2) Sublimation and imaging of the PAH-4W nano-vehicle on $\mathrm{Au}(111)$}

An atomically clean $\mathrm{Au}(111)$ surface was UHV prepared following a standard sample preparation method using several cycles of Ar+ ion sputtering and annealing up to $450^{\circ} \mathrm{C}$ [20]. PAH-4W molecules were available in the form of a green powder material. Since the molecule is quite heavy with a molar mass of $2325 \mathrm{~g} \cdot \mathrm{mol}^{-1}\left(\mathrm{C}_{184} \mathrm{H}_{116}\right)$, standard thermal sublimation using a Joule heated crucible only leads to molecular debris on the $\operatorname{Au}(111)$ surface (up to $500^{\circ} \mathrm{C}$ ). We have therefore built a specific flash evaporator using a small in section tungsten filament. A solution of the PAH-4W molecules was first prepared selecting with care a low molecular weight solvent, here dichloromethane. Then, this filament was coated with the solution using a simple drop casting technique waiting for the droplet to dry before introducing this filament in the UHV chamber of the STM. While passing a few amperes of current intensity through this filament during a fraction of seconds, the filament is heated-up instantly. Many of the adsorbed molecules are destroyed like when using a thermal crucible evaporator but a few of them survive and are released from the hot filament to the surface [21]. Including the $\mathrm{dI} / \mathrm{dV}$ mapping, all STM experiments were performed using one of the four scanning tunneling microscope heads of our ScientaOmicron low-temperature ultrahigh-vacuum STM instrument (LT-UHV 4-STM) 
operating at a base pressure of 10-10 mbar range at $5 \mathrm{~K}$ [20]. STM tips for scanning, molecular manipulations and spectroscopic measurements were prepared by electrochemical etching of a 250 micro-m tungsten wire. The tip apex was re-prepared in-situ on the $\mathrm{Au}(111)$ surface away from the PAH-4W molecules.

A typical constant current LT-UHV-STM image of the $\mathrm{Au}(111)$ reconstructed surface just after the molecules deposition is presented in Fig. 2. Many of the imaged adsorbates on this $\mathrm{Au}(111)$ surface are molecular fragments coming from PAH-4W molecules. However, a few intact PAH4W molecules can be found at step edges and rarely on terraces when stabilized by the molecular fragments. Interestingly, they were found perpendicular and always atop the step with only one of their ends, with two tert-butyl groups positioned at the border of the step edge. We have evaluated the molecule density on the $\mathrm{Au}(111)$ surface to be of about one PAH-4W molecule per $50.000 \mathrm{~nm}^{2}$, enough to perform single molecule STS spectroscopy and single molecule manipulations. Notice that according to Fig. 2, we have succeeded to tune the filament heating time and the concentration of the initial dichloromethane solution of PAH-4W in such a way that the $\mathrm{Au}(111)$ herringbone reconstruction is largely intact on large terrace, a very important point for long distance lateral manipulation. In Fig. 2, some of STM imaged debris are clearly parts of PAH-4W molecules. A detail image of one PAH-4W molecule is presented in Fig. 3a with its corresponding elastic scattering quantum chemistry (ESQC) calculated $\mathrm{dI} / \mathrm{dV}$ constant current image [22]. The four wheels, the chassis and the four tert-butyl chemical groups can be identified. The triptycene wheels have normally a larger tunnel conductance than the tert-butyl groups. But as presented in Fig. 3 insert, this is depending on the bias voltage and a relative contrast reversal between the wheels and the tert-butyls can also be observed while the central chassis is always showing a lower conductance. The surface conformation of a PAH-4W molecule adsorbed on $\mathrm{Au}(111)$ will be discussed below after detail dI/dV mappings and a few STM molecular manipulation sequences.

After this flash evaporation, the few PAH-4W molecules observed on the surface were systematically located on $500 \mathrm{~nm}$ x $500 \mathrm{~nm}$ portions of the $\mathrm{Au}(111)$ surface using the monoatomic steps configuration to record the position of each molecule on its surface portion. Here, we have taken advantage of the stability of our LT-UHV 4-STM to record their location with a sub-nanometer precision in the $\mathrm{x}$ and $\mathrm{y}$ directions on the $\mathrm{Au}(111)$ surface [20]. We have accumulated a stock of PAH-4W molecules coordinates sufficient to perform single molecule manipulation and STS tunnelling spectroscopy measurements. Fig. 2 is presenting a simple 
STM lateral manipulation of a single PAH-4W molecule out of its native step edge location to demonstrate that this PAH-4W molecule is intact and can be pushed away from this native physisorption site.

To identify the location of tunnelling conductance maxima along the PAH- $4 \mathrm{~W}$ molecular skeleton, STM dI/dV spectroscopic measurements were performed. At low bias voltage, this procedure is now a standard to identify the energy position of the molecular electronic states of the molecule close to the Fermi level when adsorbed for example on a metal surface. This procedure is also compulsory to identify the energy entry port for manipulating the molecule without any mechanical interaction between the STM tip apex and the molecule itself $[5,12$, 23]. Fig. 3(a) is presenting a typical dI/dV spectrum where two tunnelling resonances at -1.65 $\mathrm{V}$ and $-2.15 \mathrm{~V}$ and a third one at $+2.10 \mathrm{~V}$ above the bulk gold Fermi level $\left(\mathrm{E}_{\mathrm{f}}\right.$, i.e., $\mathrm{V}=0$ ) can be observed. They were recorded at slightly different locations on the same PAH- $4 \mathrm{~W}$ molecule depending on the maxima and minima of differential conductance along this molecule. Very detailed dI/dV STM maps were also recorded at these tunnelling resonance energies to visualize the spatial variation of the electron probability density distribution along a PAH- $4 \mathrm{~W}$ molecule via the tunnelling differential conductance variations along the STM scans.

According to the interpretation of previously recorded $\mathrm{dI} / \mathrm{dV}$ maps, for example on polyacene molecules like pentacene [24], the main molecular orbitals (MO) of the PAH-4W molecule contributing to the first electronic tunnel resonances at $-1.65 \mathrm{~V}$ and $+2.10 \mathrm{~V}$ are the HOMO and the LUMO respectively. Indeed, the central conjugated board of this molecule is not long enough to find for example molecular radical states contaminating the $\mathrm{PAH}-4 \mathrm{~W}$ electronic ground state [30].

For comparison, the HOMO and LUMO electronic probability density of a free (not adsorbed) PAH-4W molecule in its Fig. 1 minimum energy conformation (Fig. 1, structure i) were calculated using a semi-empirical method (MOPAC [25]) and are presented in Fig. 3c. Because of the molecular design with its four wheels and also its tert-butyl-groups bound to the central curved chassis via sigma-bonds, the electronic states near the HOMO-LUMO gap are well separated in energy between the wheels and the tert-butyl mono-electronic states. The HOMO and LUMO of a free PAH-4W molecule are mainly the chassis MO characterized by extended central $\pi$ molecular orbitals on the chassis. According also to those calculations, the next set of PAH-4W MO and after the chassis ones, can be attributed to the wheels $\pi$ MOs. 
Following the experimental recorded $\mathrm{d} \mathrm{I} / \mathrm{dV}$ maps, the reverse is observed on the surface for the ground state tunnelling resonance (and below) i.e. the Fig. $3 \mathrm{~b} \mathrm{dI} / \mathrm{dV}$ maps recorded at the -1.65 $\mathrm{V}$ and $-2.15 \mathrm{~V}$ electronic resonances are showing a large four wheels MO contribution to the

tunnel differential conductance as compared to the chassis. As expected, the first tunnelling resonance above $\mathrm{E}_{\mathrm{f}}(\mathrm{LUMO})$ is still located on the chassis. For the ground state resonance (HOMO), this reversal between the $\mathrm{dI} / \mathrm{dV}$ resonances (identified using the $\mathrm{dI} / \mathrm{dV}$ maps) and the Fig. 3c MO electron probability density maps is coming from the PAH-4W molecule conformation on the $\mathrm{Au}(111)$ surface with its chassis to surface distance certainly much larger than the triptycene wheel low phenyls distance to the surface. As a consequence, the wheels electronic states contributing to the PAH-4W electronic states move up in energy towards the ,chassis electronic state energy position because of their electronic interaction with the $\mathrm{Au}(111)$ surface. This was already observed on a molecule with a central axle terminated by a triptycene wheel at each extremity [18]. As a result, the first resonance recorded at $-1.65 \mathrm{~V}$ can be attributed to electronic contributions coming both from the chassis and wheels electronic states lying after molecular adsorption in the same energy window. Furthermore, since the height of the chassis relative to the surface is certainly much larger than the triptycene wheel down phenyls distance to this surface (See Fig.7), the wheels electronic states are contributing more than the chassis ones to the $\mathrm{dI} / \mathrm{dV}$ map recorded at $-1.65 \mathrm{~V}$. The resonance observed at $\mathrm{V}=-2.15 \mathrm{~V}$ also originates from the wheels, as confirmed by ESQC calculated constant current STM image.

This $\mathrm{dI} / \mathrm{dV}$ interpretation indicates that the stable conformation of a PAH-4W molecule on $\mathrm{Au}(111)$ brings the four wheels down and the four tert-butyl chemical groups up relative to the surface because of the same STM contrast of those tert-butyl groups (See the Fig. 3a ESQC calculated image for the surface conformation with the wheels down and the tert-butyl groups up as in Fig. 7a). This will be discussed further below. More important is also the fact that the resonances at $-1.65 \mathrm{~V}$ and $-2.15 \mathrm{~V}$ are mainly located on the wheels. On the PAH-4W molecular skeleton, it indicates the locations where to pulse the bias voltage to bring some inelastic energy inside the molecule for a possible step by step STM manipulation along the surface without any mechanical contact of the PAH-4W molecule with the tip apex.

\section{3) Single molecule manipulations}


Two modes of STM single molecule manipulation were explored, first by pulsing the STM bias voltage for a few seconds at a specific location on the PAH-4W molecule and second by pushing this molecule using the tip apex.

\section{1) Voltage pulsing mode}

For the pulsing mode, the tip apex was positioned on a wheel and the bias voltage pulsed down to $-2.0 \mathrm{~V}$ and up to $+3.2 \mathrm{~V}$. A $-2.0 \mathrm{~V}$ pulse was high enough to access the first oxidation state of a wheel (See Fig. 3). In contrast, with a $+3.2 \mathrm{~V}$, the wheels first virtual reduced states are very difficult to reach since pulsing with a bias voltage just above $+3.2 \mathrm{~V}$ led the targeted wheel to break and detach from the chassis.

Incidentally and among the different conformations of the PAH-4W molecule identified in Fig.1, conformer (ii) was also observed from time to time on the surface. Figure 4 is presenting an example of such a conformer where the bottom-left tert-butyl is up according to Fig. 1(ii). But on the surface this bottom-left wheel will remain almost at the same height as compared to the three others due to the interaction with the surface. This PAH-4W conformation can be considered at first as a good candidate for monitoring the response of a wheel to the voltage pulse. When the bias voltage is applied with the tip apex positioned on the edge of this upwheel, it starts to fluctuate randomly in orientation relative to its rotation axle as observed in the current intensity time dependent signal presented in Figure 4(b). The current intensity is switching between $10 \mathrm{pA}$ and $85 \mathrm{pA}$, corresponding alternatively and randomly to two molecular conformations of the triptycene wheel: respectively one phenyl pointing down towards the surface and one phenyl pointing up towards the tip apex. This indicates that a curved chassis can lead to an up-wheel conformation according to Fig. 1(ii). Furthermore, the switching from a down (Fig. 1 i) to an up-wheel (Fig. 1 ii) conformation requires to pass over a large energy barrier in the PAH-4W electronic ground state. Unfortunately, it cannot be performed on the wheels sitting down corresponding to the main PAH-4W conformation on the surface (Figure 7a). This comes from the fact that the consequent vibrational energy increase will mainly remain on the chassis. Here, the corresponding Fig. $3 \mathrm{~b} d \mathrm{~d} / \mathrm{dV}$ maps are indicating four very local conductance maxima at the centre of the chassis with no extension towards the wheels. This indicates that contrary to what was observed in the case of a chassis equipped with four switchable legs [9] and even with a curved chassis, the four wheels cannot be switched at the same time away from the surface and this will be incompatible with the possible accessible 
conformation of the chassis (Fig. 1(iii)) even on the surface. Furthermore, we do not have identified yet the tip apex position on the chassis (or on a given wheel) to trigger the corresponding two up-wheel chassis conformation change in the PAH-4W excited states accessible by a bias voltage pulse.

\section{2) Mechanical manipulation mode}

For the pushing mode and in a now standard mechanical molecular manipulation procedure [26], a PAH-4W molecule can be step by step manipulated on the Au(111) surface. A typical constant current manipulation signal is presented in Fig. 5 together with the tip trajectory. It was recorded during the manipulation sequence indicated also on the STM images before and after the manipulation. To record this Fig. 5 molecular manipulation signal, the molecule was initially found in its native as deposited step edge conformation and the tip apex was initially positioned on the centre of its chassis. The first $2 \mathrm{~nm}$ of the manipulation sequence corresponds to the detaching of the PAH-4W molecule from its step edge (Fig. 5(i)). It is generally a quite erratic sequence because the molecular mechanics of this detachment is dependent on the tip apex end atomic structure and because a step edge is not a very regular support for the molecule itself especially for a four wheels molecule. Then, from $2.0 \mathrm{~nm}$ to $3.4 \mathrm{~nm}$ and from $3.7 \mathrm{~nm}$ to $5.2 \mathrm{~nm}$ (Fig. 5, (ii) and (iv)), the constant current manipulation signal in this pushing mode is quite regular. In the Fig. 5 reported manipulation sequence, it is coming from the bottom-left wheel with respect to the direction of movement. Such types of constant current manipulation signals are resulting from the through molecule tunnel current intensity variation as a function of the detail of the mechanical deformation of the molecule during its manipulation. It had already been used to determine detailed intramolecular conformation changes in particular with a molecule equipped with four legs [27] and with a molecule equipped with two triptycene wheels [18].

The manipulation signal reported in Fig. 5 between $2.0 \mathrm{~nm}$ and $3.4 \mathrm{~nm}$ and between $3.7 \mathrm{~nm}$ and $5.2 \mathrm{~nm}$ is very similar to the one recorded in this last case when the wheels were not rotating [18]. This also indicates that after the first manipulation sequence (where the molecule had been detached from its step edge) the tip apex slides along the chassis to reach in this case the bottomleft wheel. The interruption of the regularity of the pushing mode manipulation signal between $3.4 \mathrm{~nm}$ and $3.7 \mathrm{~nm}$ is coming from the herringbone ridge. In this case, wheel after wheel, the molecule had to pass over this $\mathrm{Au}(111)$ surface reconstruction which is known to be quite difficult to pass over with a moderate mechanical push [28]. The oscillation period of the 
manipulation signal during the regular parts is about $0.28 \mathrm{~nm}$ i.e. close to the 0.288 nearest neighbour interatomic distance on the $\mathrm{Au}(111)$ surface. Notice that at the end of this Fig. 5 manipulation sequence, one of the four wheels attached to its triple bond axle was always found under the final tip position. This confirms that the largest mechanical interaction between the tip and the molecule is located in this part of the molecule and that the lateral motion of the tip during a constant current manipulation sequence leads the tip apex to reach this specific intramolecular location.

Fig. 6 is presenting another example of a PAH-4W molecule manipulation sequence performed in five steps continuously and without any STM imaging during this sequence. Characteristic behaviours appear along this sequence at $3 \mathrm{~nm}$ and at $8 \mathrm{~nm}$ tip apex positions starting from the presented initial tip apex position on the chassis. At these tip locations, the manipulation signal suddenly jumps up, remains up for about $2 \mathrm{~nm}$ with a typical manipulation signature (see Fig. 6 inserts) and then drops down to its previous relative $\Delta z$ tip height value. We interpret this manipulation signature as a rotation of the wheels while passing over the herringbone reconstruction as it was observed recently by pushing on a "dimer of triptycene wheels" molecule [18]. It also indicates that before the start of Fig. 6 manipulation sequence, two phenyl rings out of the three on each triptycene wheel were in interaction with the $\mathrm{Au}(111)$ surface. It was the minimum energy conformation of the PAH-4W molecule before the Fig. 6 sequence (see the discussion section below). The wheel axle height variation during a wheel rotation is about 220 pm (Fig.6) and the recorded manipulation signal jump up at $3 \mathrm{~nm}$ and $8 \mathrm{~nm}$ is about $120 \mathrm{pm}$. A third jump in the manipulation signal seems to have occurred at $13 \mathrm{~nm}$ but has been obscured. This can be due to either a tip apex condition change or a wrong tip positioning for such a wheel rotation sequence, because of a wrong orientation of the PAH-4W molecule relative to the underneath herringbone reconstruction orientation. This results in a fast drop of the manipulation signal at the beginning of fourth manipulation step.

The PAH-4W can also be manipulated over very long distances in a pushing mode with the tip apex remaining in between the four wheels. We have succeeded in runs of about $25 \mathrm{~nm}$. The difficulty in such long pushing manipulation sequences is the successive passage over a large number of herringbone reconstructions since already passing a succession of three reconstructions is delicate as presented in Fig. 6. Notice that without success, we have also tried to observe in the manipulation signal the rotation of the wheels during a passage over an $\mathrm{Au}(111)$ mono-atomic step in the constant current manipulation. Here, it was found difficult 
with a clear signature in the variation of the tunnel current to record a regular and classical mechanical motion of the PAH-4W molecule passing wheel after wheel over an $\mathrm{Au}(111)$ step edge $0.22 \mathrm{~nm}$ in height. The PAH-4W molecule is not a rigid object with rigid rotating wheels and its classical mechanical motion is difficult to define [29]. All the deformed parts of the molecule during its collision with the step edge are contributing to the tunneling current variations.

\section{4) Discussions}

On a surface, a long range single molecule nanocar run is dependent on the accessible conformations of the molecule while adsorbed on its supporting surface. Those conformations are determined by the multi-dimensional electronic ground state potential energy surface (PES) of this molecule completed for a controlled drive on this surface by the manifold topology of the low lying electronic excited states PES. The surface ground state conformations of molecule nano-vehicles equipped with lateral holders are also very dependent on the competition between the chassis and the holder's interactions with the supporting surface. The curved chassis of the PAH-4W molecule studied here was designed to master this competition as compared to a planar chassis [8]. On the supporting $\mathrm{Au}(111)$ surface, the PAH-4W chassis is constrained to curve up at its extremities letting the four triptycene wheels to be less interacting with the surface than in the case of a planar chassis. Experimental constant current STM images and molecular mechanics optimization on surface are confirming how the chassis effectively curves up at its two ends in its minimal energy conformation as presented in Fig 7a. As a consequence, the STM contrast delivered by the four tert-butyl end groups is smaller than the STM contrast given by the four triptycene wheels (See Fig. 3). This curved conformation is still preserving enough the interactions between the wheels and the surface for them to be able to turn while passing over the $\mathrm{Au}(111)$ herringbone reconstructions as observed experimentally in Fig. 6. In the other possible and stable conformation calculated for the PAH-4W molecule on the $\mathrm{Au}(111)$ surface, the four tert-butyl groups are down to the surface and the centre of the chassis is curved up (see Fig. 7b). In this conformation, the four triptycenes are too far away from the surface to be stabilized and will rotate freely with no interaction with this surface. The formal Fig. 7a conformation is confirmed by ESQC STM constant current image calculations performed exactly with this conformation. The latter Fig. $7 b$ conformation was not observed on the $\mathrm{Au}(111)$ surface. 
In the Fig. 7a minimal energy surface conformation, the activation of a lateral motion of the PAH-4W molecule using a bias voltage pulse was not observed. This activation would require to switch up the conformation of each phenyl holding the triptycene wheel axle in such a way to make free the rotation of each wheel relative to the surface, but with the wheels (and not the four tert-butyls) still holding the chassis on the surface. Such switching event was not observed. With a positive voltage pulse larger than $+3.2 \mathrm{~V}$, it was possible to break one wheel out of the chassis. The switching up of one (or more) phenyl holding a wheel imposes too much a constraint to the chassis. Furthermore, and as observed, such a conformation is not accessible energetically on the low lying excited states PES of the PAH-4W molecule adsorbed on the $\mathrm{Au}(111)$ surface. A bias voltage pulse applied at the center of the chassis may have been an answer to activate this switching because of the peculiar localization on the chassis of the $+2.1 \mathrm{~V} \mathrm{dI} / \mathrm{dV}$ resonance. Unfortunately, the provided energy is not large enough to trigger the simultaneous switching of the four phenyl groups holding the wheels. Even if the reactive path on the corresponding excited states PES was existing, the sequence of switching events in time for the PAH-4W molecule to move classically in one direction would have to be encoded in those potential energy surface.

\section{5) Conclusions}

A four wheels molecule nano-vehicle with a curve central molecular chassis was deposited on an $\mathrm{Au}(111)$ surface and imaged at low temperature using a scanning tunneling microscope. The consequent moderate interactions of those four wheels with the $\mathrm{Au}(111)$ surface was confirmed. Along the molecular chassis, constant current $\mathrm{dI} / \mathrm{dV}$ maps were recorded to locate the possible energy entry port for a controlled manipulation of the molecule. The switching of the phenyl holding a wheel to the chassis was not observed to trigger a lateral molecular motion by inelastic tunneling effect through the molecule when the STM tip apex is located on this phenyl. A standard STM tip mechanical manipulation of the molecule was used to confirm how the wheels can step by step rotate while passing over an $\mathrm{Au}(111)$ surface native herringbone reconstruction. This rotation was time-dependent recorded by following in real time the variation of the tunneling current intensity during such a manipulation. New molecules must now be designed in such a way that the sequence of wheel rotations be defined on the profile of potential energy surface of the excited states to be able to drive a molecule-vehicle by an inelastic tunneling effects starting from the ground electronic states of the molecule nano-vehicle. 
Acknowledgements: This work was supported by the University Paul Sabatier (Toulouse, France), the Centre National de la Recherche Scientifique (CNRS), the Agence Nationale de la Recherche (ANR) (ACTION project ANR-15-CE29-0005). This work has received funding from the European Union's Horizon 2020 research and innovation program under the project MEMO, grant agreement No 766864. We also would like to thank Peugeot, Actia and Air Liquide for their kind and financial supports during this work and J. Bonvoisin for discussion about the free conformation of the PAH-4W molecule. 


\section{References:}

[1]: C. Joachim, G. Rapenne, ACS Nano, 7, 11 (2013).

[2]: C. Joachim, H. Tang, F. Moresco, G. Rapenne, G. Meyer, Nanotechnology, 13, 330 (2002).

[3] Jimenez-Bueno, G.; Rapenne, G.Tetrahedron Lett. 2003, 44, 6261.

[4] Shirai, Y.; Osgood, A. J.; Zhao, Y.; Kelly, K. F.; Tour, J. M. Nano Lett. 2005, 5, 2330.

[5]: W.H. Soe, Y. Shirai, C. Durand, Y. Yonamine, K. Minami, X. Bouju, M. Kolmer, K. Ariga, C. Joachim, W. Nakanishi, ACS Nano, 11, 10357 (2017).

[6]: Y. Shirai, A. Osgood, Y. Zhao, Y. Yao, L. Saudan, H. Yang, C. Yu-Hung, L.B. Alemany, T. Sasaki, J.F. Morin, J. Tour, J. Am. Chem. Soc., 128, 4854 (2006).

[7]: G. Rapenne, Org. Biomol. Chem., 3, 1165 (2005).

[8]: G. Rapenne, G. Jimenez-Bueno, Tetrahedron, 63, 7018 (2007).

[9]: T. Kudernac, N. Ruangsupapichat, M. Parschau, B. Maci, N. Katsonis, S.R. Harutyunyan, K.H. Ernst, B.L. Feringa, Nature, 479, 208 (2011).

[10]: P.T. Chiang, J. Mielke, J. Godoy, J.M. Guerrero, L.B. Alemany, C.J. Villagomez, A. Saywell, L. Grill, J.M. Tour, ACS Nano, 6, 592 (2012).

[11]: J. Godoy, G. Vives, J.M. Tour, ACS Nano, 5, 85 (2011).

[12]: R. Pawlak, T. Meier, N. Renaud, M. Kisiel, A. Hinaut, T. Glatzel, D. Sordes, C. Durand, W.H. Soe, A. Baratoff, C. Joachim, C. E. Housecroft, E. C. Constable, E. Meyer, ACS Nano, 11, 9930 (2017).

[13]: H.P. Jacquot de Rouville, R. Garbage, R.E Cook, A.R. Pujol, A.M. Sirven, G. Rapenne, Chem. Eur. J., 18, 3023 (2012).

[14]: T. Sasaki, J.M. Tour, Tetrahedron Lett., 48, 5821 (2007).

[15]: G.J. Simpson, V. Garcia-Lopez, P. Petermeier, L. Grill, J. M. Tour, Nature Nano, 12, 604 (2017).

[16]: L. Gross, K.H. Rieder, F. Moresco, S. Stojkovic, A. Gourdon, C. Joachim, Nature Mat., 4, 892 (2005).

[17]: L. Gross, F. Moresco, C. Wang, G. Rapenne, G. Jimenez, C. Joachim, Surf. Sci. Lett., 584, L153 (2005).

[18]: L. Grill, G. Rapenne, S. Stojkovic, X. Daya, C. Joachim, F. Moresco, Nature Nano, 2, 95 (2007)

[19]: A. Moscatelli, Nature Nano, 12, 597 (2017). 
[20]: J. Yang, D. Sordes, M. Kolmer, D. Martrou, C. Joachim Eur. Phys. J. AP, 73, 10702 (2016).

[21]: T. Zambelli, Y. Boutayeb, F. Gayral, J. Lagoute, N.K. Girdhar, A. Gourdon, S. Gauthier, M.-J. Blanco, J.-C. Chambron, V. Heitz, J.-P. Sauvage, Int. Jour. Nanoscience, 3, 331 (2004).

[22]: P. Sautet, C. Joachim, Chem. Phys. Lett., 185, 23 (1991).

[23]: M. Lastapis, M. Martin, D. Riedel, L. Hellner, G. Comtet, G. Dujardin, Science, 308, 1000 (2005).

[24]: W.H. Soe, C. Manzano, A. De Sarkar, N. Chandrasekhar, C. Joachim Phys. Rev. Lett., 102, 176102 (2009).

[25]: J.J.P. Stewart, J. Comp. Chem., 10, 209 (1989) and 10, 221 (1989).

[26]: T.A. Jung, R.R. Schlittler, J.K. Gimzewski, H. Tang, C. Joachim, Science, 271, 181 (1996).

[27]: F. Moresco, G. Meyer, K.H. Rieder, H. Tang, A. Gourdon, C. Joachim, Phys. Rev. Lett., 87, 088302 (2001).

[28]: C. Manzano, W.H. Soe, H.S.J. Wong, F. Ample, A. Gourdon, N. Chandrasekhar, C. Joachim, Nature Mat., 8, 576 (2009).

[29]: H. Tang, M.T. Cuberes, C. Joachim, J.K. Gimzewski, Surf. Sci., 386, 115 (1997).

[30]: Y. Yang, E.R. Davidson, W. Yang, PNAS, 113, E5098 (2016). 

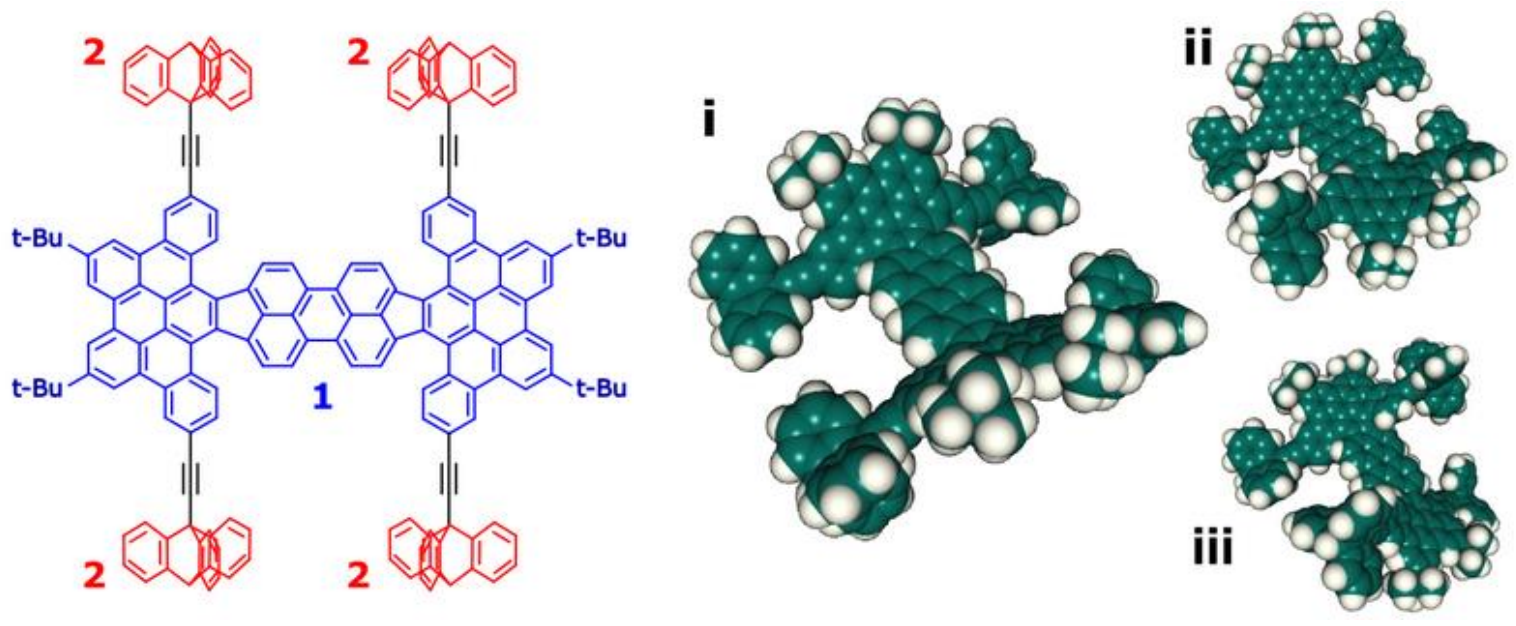

Figure 1: The chemical skeleton (left) and three possible optimized conformations of the free PAH-4W molecule using the MOPAC package [27]. The central molecular chassis is in blue and the 4 triptycene molecular wheels in red (the chemical group marked with a " 2 "). Optimized structure $\mathbf{i}$ is showing how the 4 triptycene wheels are pushed down the chassis through a steric saddle shape chassis structure, with 4 wheels down and 4 tert-butyls groups up, due to deformation induced by the presence of two five-membered rings on the central part of the chassis. In the case of other optimized structure ii (or iii, not found experimentally), the deformation occurs in 3 wheels and 1 tert-butyl group (2 wheels and 2 tert-butyls groups respectively) up and 1 wheel and 3 tert-butyls groups ( 2 wheels and 2 tert-butyls groups) down. 


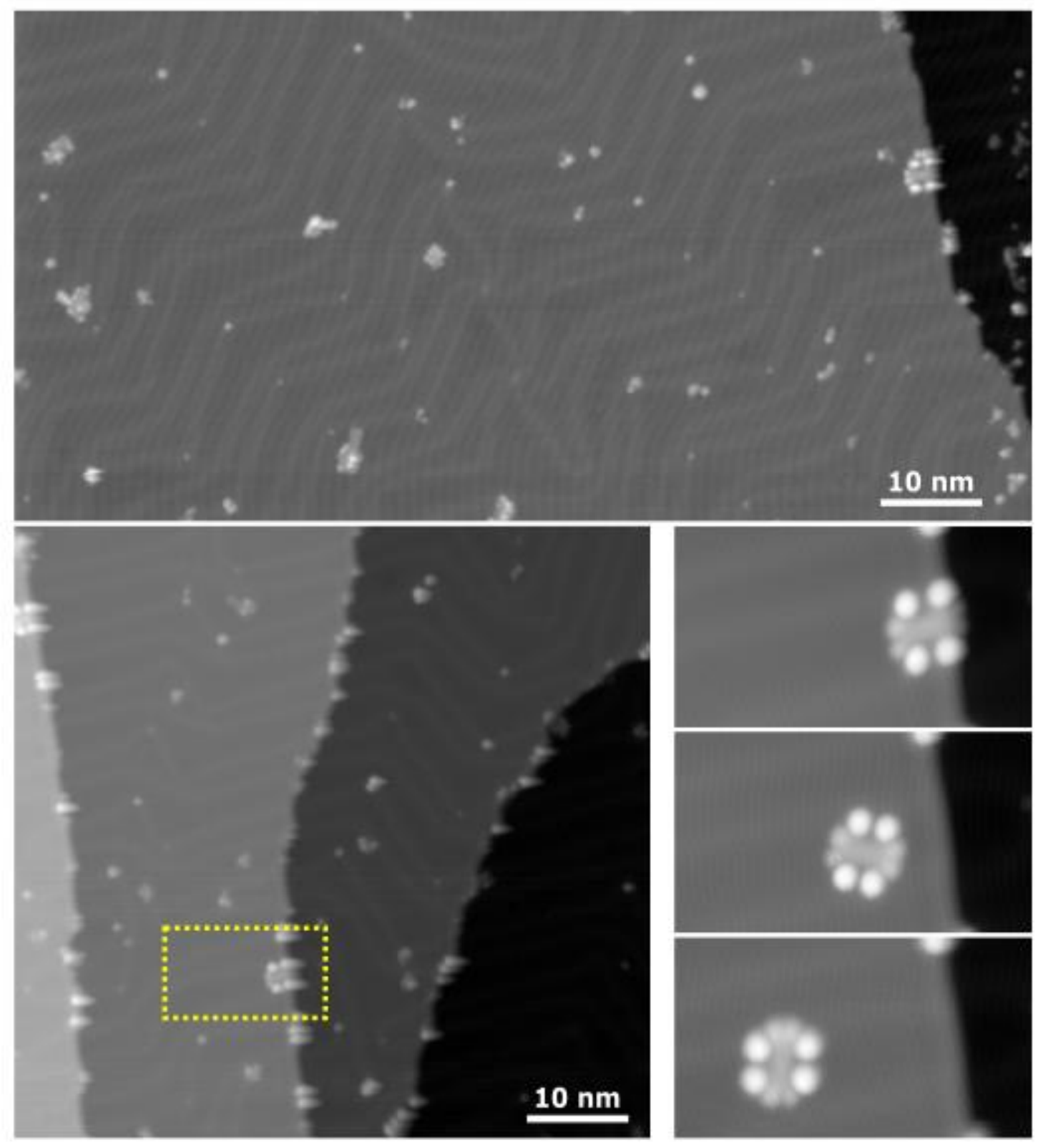

Figure 2: Typical STM images just after the molecule sublimation process. Since intact PAH$4 \mathrm{~W}$ molecules are mainly stabilized at the step edges, the probability of finding molecules decreases inversely to the terrace width. Only one intact molecule was found in top STM image area $\left(1\right.$ molecule per $\left.5000 \mathrm{~nm}^{2}\right)$ by comparison with two intact molecules in bottom left image (1 molecule per $1800 \mathrm{~nm}^{2}$ ). Bottom right image series shows a sequence of 3 lateral manipulations to detach the molecule from its initial step edge. First image of the series is corresponding to the dotted area of the large bottom left STM image. All images were taken with tunnel current $\mathrm{I}=10 \mathrm{pA}$ and bias voltage $\mathrm{V}=200 \mathrm{mV}$. Lateral mechanical manipulation condition for the right series: $\mathrm{I}=10-20 \mathrm{nA}$ with $\mathrm{V}=50 \mathrm{mV}$. 

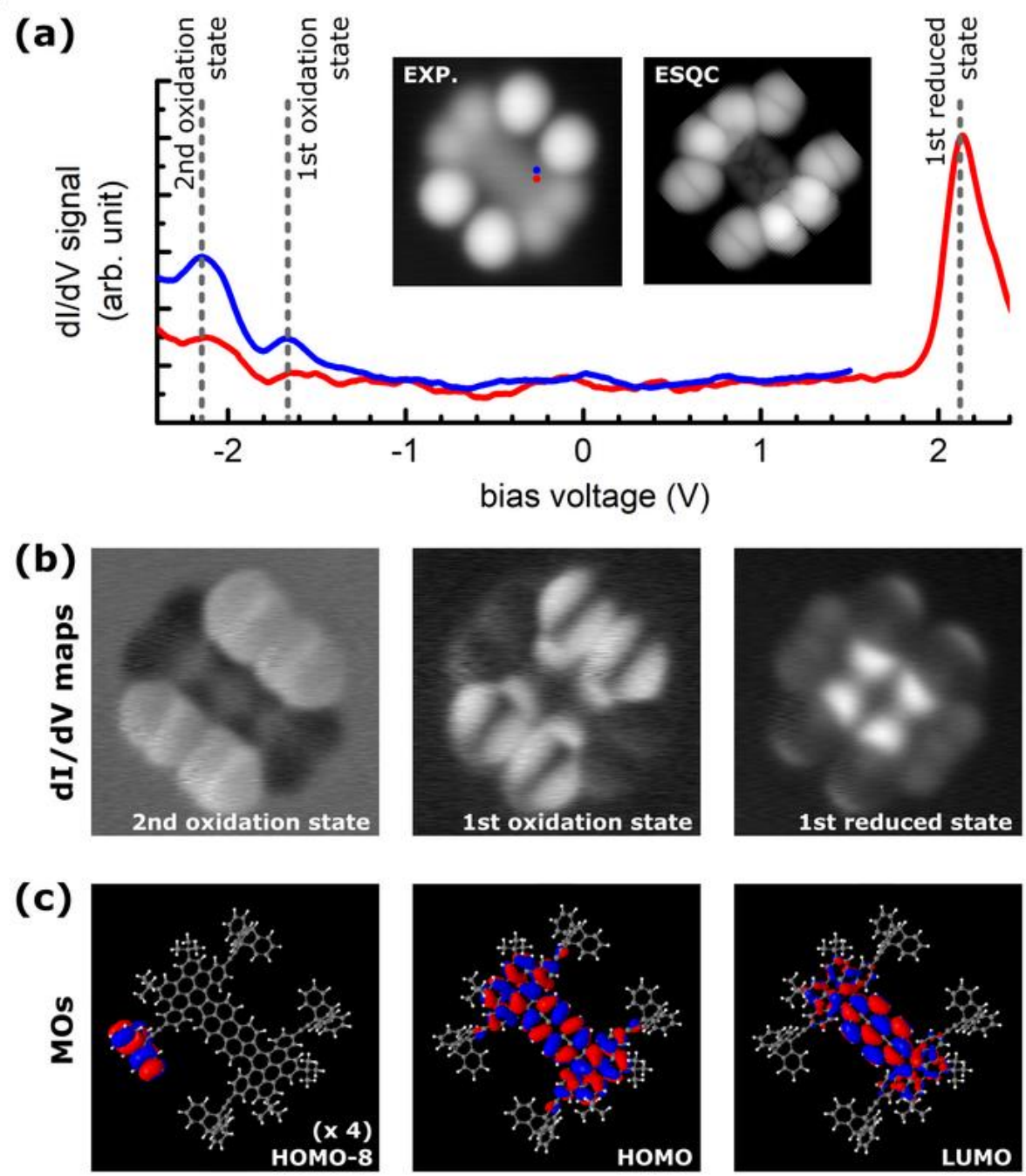

Figure 3: (a) Typical scanning tunneling $\mathrm{dI} / \mathrm{dV}$ spectra recorded at slightly different tip locations on the molecule chassis but near the wheel. The corresponding low voltage experimental image is presented also in (a) with the location of the tip during spectroscopic measurements indicated as blue and red spots (corresponding to the blue and red curves, respectively). The corresponding $\mathrm{dI} / \mathrm{dV}$ ESQC calculated image is presented in (a). Three distinct peaks, one at positive as first virtual reduced state and two at negative bias voltage as first and second virtual oxidation states, are observed and identified by vertical dotted lines. (b) The experimental differential conductance $(\mathrm{dI} / \mathrm{dV})$ maps recorded at the bias voltage identified in (a) (they realize a projection of the PAH- $4 \mathrm{~W}$ electronic probability density of these molecular electronic states on a two dimensional plane). In order to determine which mono-electronic molecular states are composing the experimentally observed electronic probability density maps, molecular orbitals of the free PAH-4W are represented at the bottom row (c) and were calculated using the semi-empirical PM7-MOPAC package [27]. Notice that the electron density of not only HOMO and LUMO but also up to HOMO-7 crowds into the chassis. The first mono-electronic state located on a wheel is arising at HOMO-8. The 4 wheel states are electronically separated from each other and can be found between HOMO-8 to HOMO-11. Only one was represented in (c). Images sizes are $4 \mathrm{~nm}$ x $4 \mathrm{~nm}$ for topographic image and $5 \mathrm{~nm}$ $\mathrm{x} 5 \mathrm{~nm}$ for $\mathrm{d} \mathrm{I} / \mathrm{dV}$ maps. 

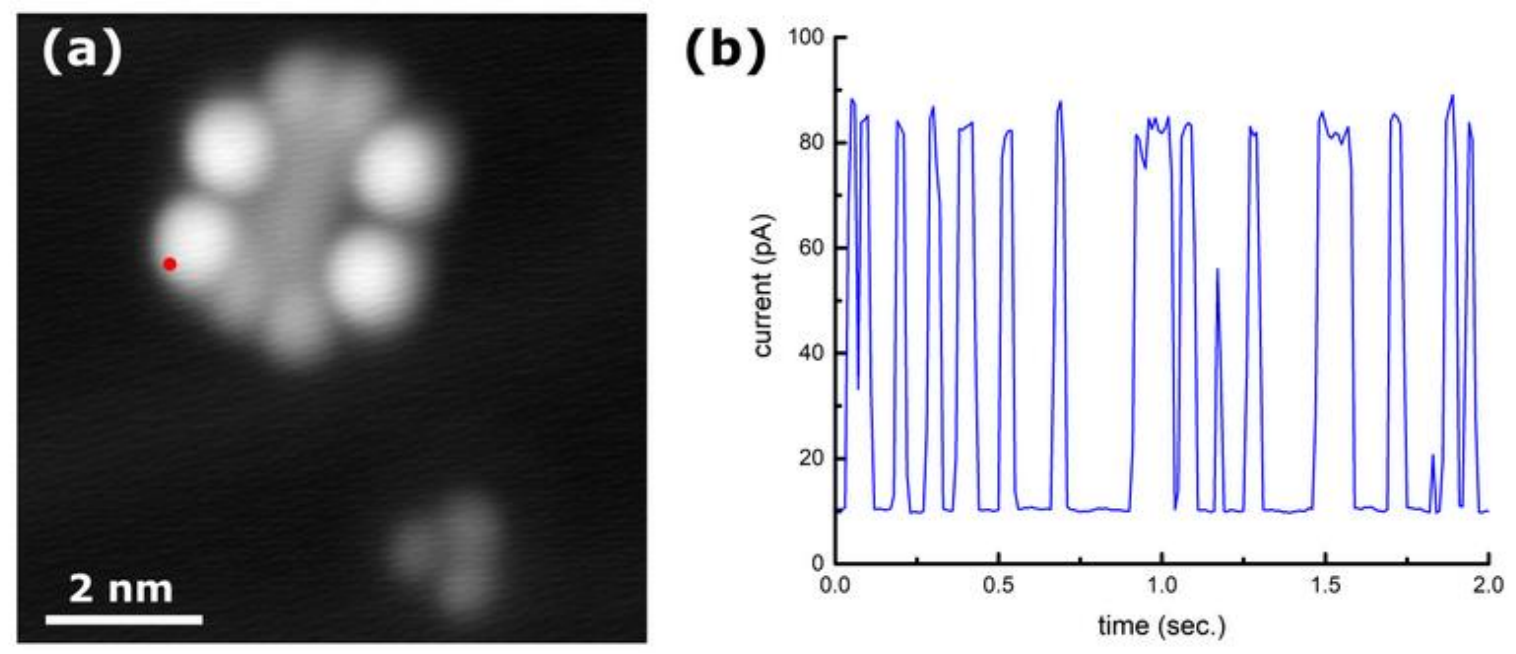

Figure 4: (a) An STM image of the PAH-4W having the conformation presented in Fig. 1(ii). Here the bottom-left tert-butyl group shows a relatively smaller lobe than the other three tertbutyls because it is down sideways and is related to the lift up of the neighboring bottom-left wheel. No significant difference in contrast between this wheel and the other three in the constant current image. (b) A real time recording of the tunneling current at $\mathrm{V}=3.2 \mathrm{~V}$ measured for the tip located on the lifted up wheel edge indicated by the red dot in (a). The current is fluctuating between $10 \mathrm{pA}$ and $85 \mathrm{pA}$ because of the random oscillations of this wheel. (Constant current image (a): $\mathrm{I}=10 \mathrm{pA}$ and bias voltage $\mathrm{V}=200 \mathrm{mV}$ ). 

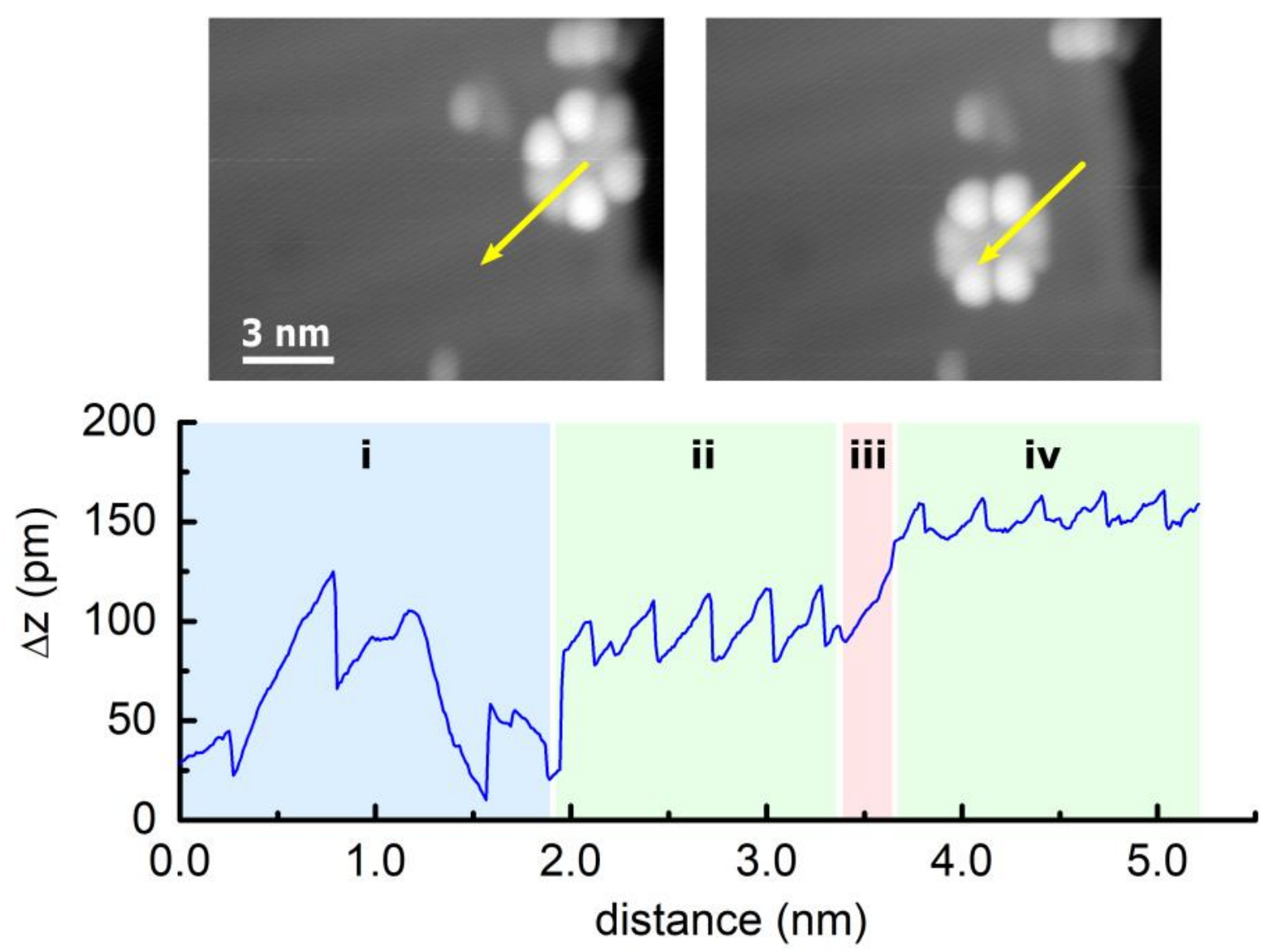

Figure 5: Typical tip height variations during an STM constant current lateral mechanical molecule manipulation with tip trajectory indicated on the images before (left) and after (right) manipulation. The manipulation signal can be separated in four sequences; (sequence $\mathbf{i}$, blue background) $0.0 \rightarrow 2.0 \mathrm{~nm}$, the molecule was pulled apart from step edge leading to the highest mechanical interaction location of the tip apex with the molecule. This is the location of the corresponding wheel shaft, on the bottom-left wheel in this case. (Sequence ii, green) $2.0 \rightarrow 3.4$ $\mathrm{nm}$, the molecule was manipulated by normal mechanical pushing mode characterized by a 0.28 $\mathrm{nm}$ period signal, which coincides approximately with the nearest neighbour interatomic distance on the $\mathrm{Au}(111)$ surface. (Sequence iii, pink) $3.4 \rightarrow 3.7 \mathrm{~nm}$, the wheel is interacting with the herringbone ridge and is responsible for the main manipulation signal jump. (Sequence iv, green) $3.7 \rightarrow 5.2 \mathrm{~nm}$, the molecule was manipulated the same way as compared to sequence ii (Constant current images: $\mathrm{I}=10 \mathrm{pA}$ and bias voltage $\mathrm{V}=200 \mathrm{mV}$ ). 

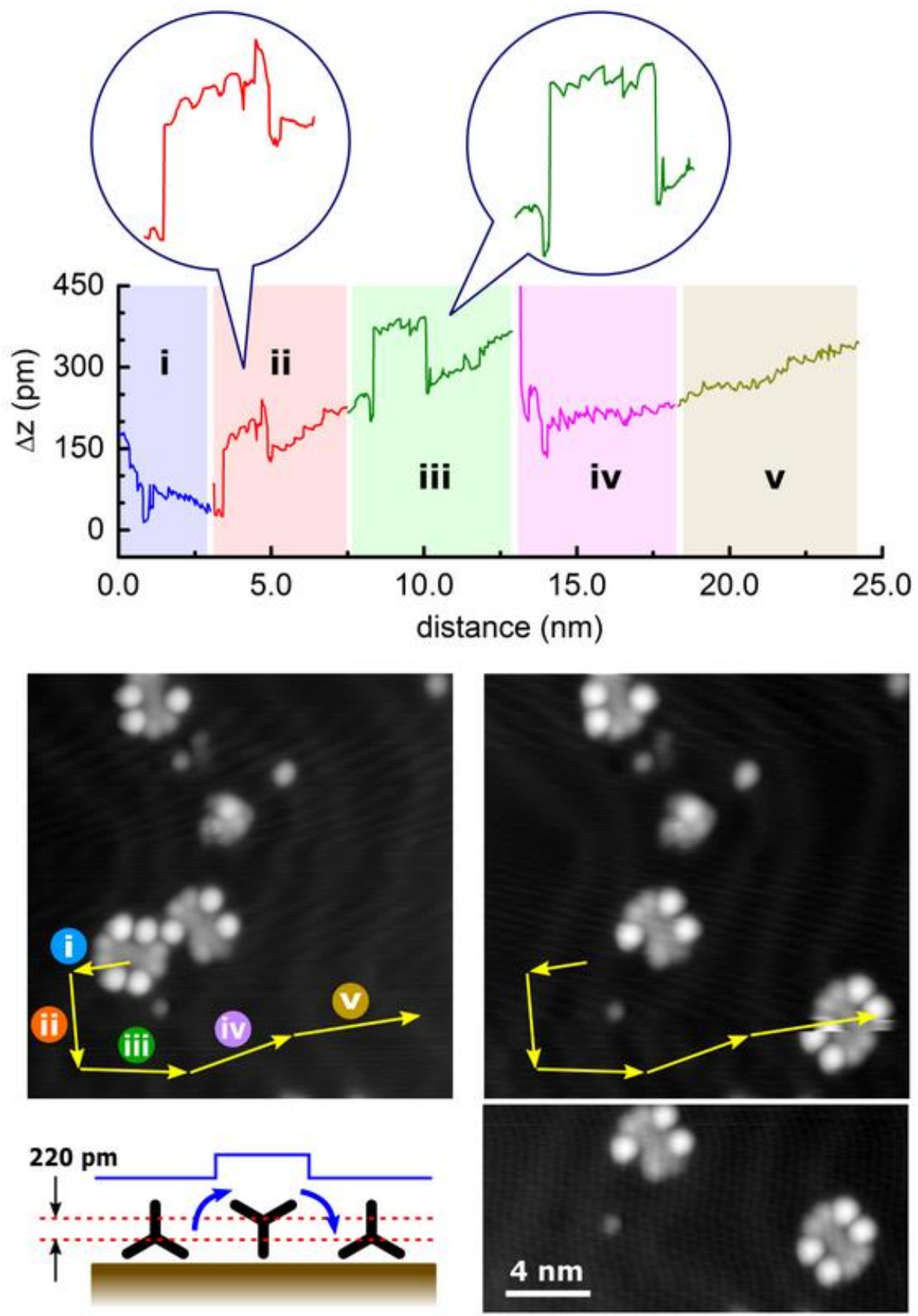

Figure 6: Another example of a PAH-4W mechanical molecule manipulation. Here the manipulations were performed five times in a row without any STM imaging during this manipulation sequence. The first $2 \mathrm{~nm}$ of the manipulation signal are essentially identical to the first example, that is, a complete $\mathrm{PAH}-4 \mathrm{~W}$ molecule was detached from a stable adsorption site created with a broken molecule. From $2.0 \mathrm{~nm}$ to $24.5 \mathrm{~nm}$, the molecule was pushed by the tip apex in a normal manipulation mode except five discontinuous points at 3, 5, 8, 10, and $13 \mathrm{~nm}$. The first and third jumps up of the manipulation signal (at 3 and $8 \mathrm{~nm}$, respectively) are the same event and are corresponding to a wheel rotation as illustrated in the left-half of the bottomleft diagram, here the wheel axis height changes during rotation. The second and fourth jumps up of this manipulation signal (at 5 and $10 \mathrm{~nm}$, respectively) are also similar events as the first (third) one. In this case, the wheel rotates like with a decrease of the wheel axis height as illustrated in the second half of the diagram. The last one is obscured by either a tip apex condition change or a wrong tip positioning (Constant current images: $\mathrm{I}=10 \mathrm{pA}$ and bias voltage $\mathrm{V}=200 \mathrm{mV}$ ). 


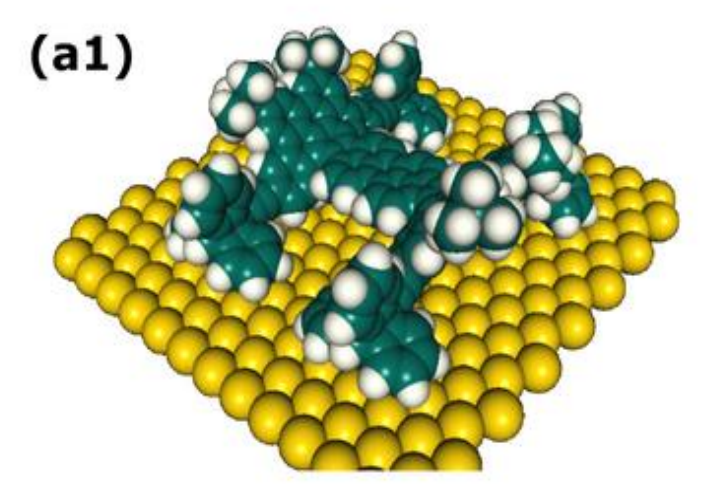

(a2)

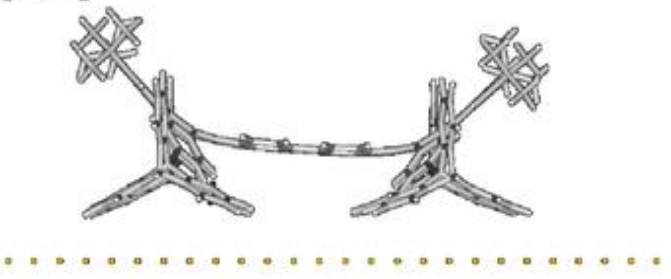

(a3)

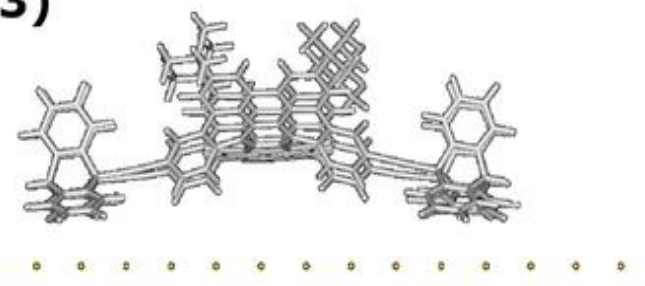

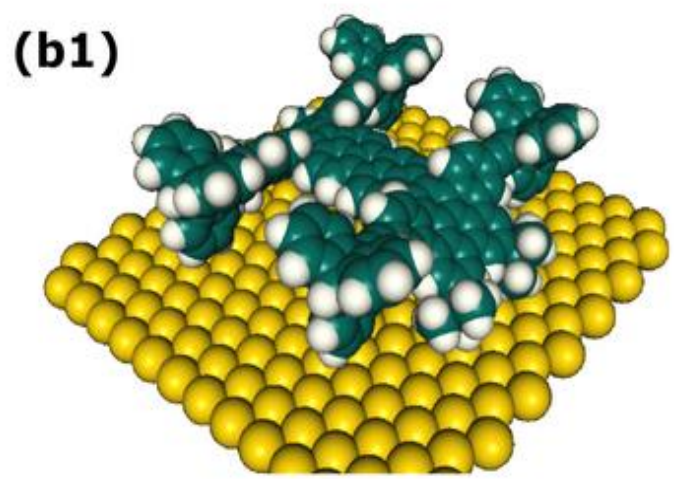

(b2)

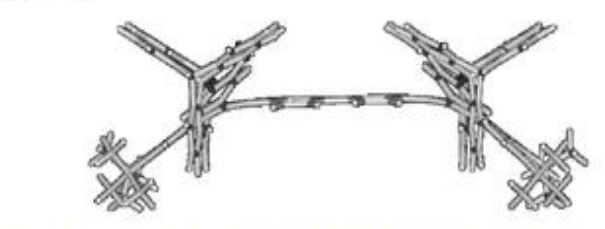

(b3)

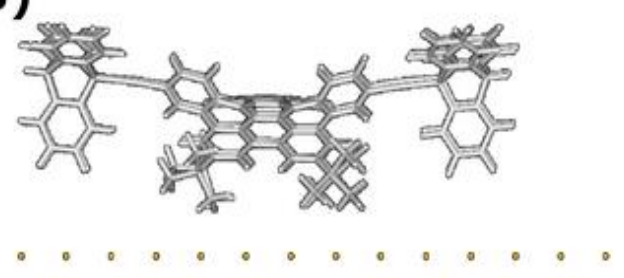

Figure 7: The two different PAH-4W surface main minimum energy molecular conformations (a) and (b) calculated using the ASED+ [32] semi-empirical molecular mechanics approach on the fcc $\mathrm{Au}(111)$ surface. The central curved down chassis conformation (a) with its characteristics up ter-butyls (a2) is the minimum energy conformation. It was used for the $\mathrm{d} / \mathrm{dV}$ constant current ESQC STM image calculation (see Fig. 3). In this conformation, the four triptycene wheels are gently deformed from $120^{\circ}$ in the gas phase to $134^{\circ}$ as exemplified in (a2). The curved up chassis conformation (b) detailed in (b2) was never observed experimentally. Here the 4 wheels are maintained far away from the surface by the chassis (b3). According to ASED+, the energy difference between (a) and (b) is $0.138 \mathrm{eV}$. 